\title{
Spectropolarimetry of Type Ibc Supernovae
}

\author{
Masaomi Tanaka ${ }^{1}$, Koji S. Kawabata ${ }^{2}$, Takashi Hattori ${ }^{3}$, \\ Paolo A. Mazzali ${ }^{4,5}$, Kentaro Aoki ${ }^{3}$, Masanori Iye ${ }^{1}$, Keiichi Maeda ${ }^{6}$, \\ Ken'ichi Nomoto ${ }^{6}$, Elena Pian ${ }^{7}$, Toshiyuki Sasaki ${ }^{3}$, \\ and Masayuki Yamanaka ${ }^{2,8}$ \\ ${ }^{1}$ National Astronomical Observatory, Mitaka, Tokyo, Japan \\ email: masaomi.tanaka@nao.ac.jp \\ ${ }^{2}$ Hiroshima Astrophysical Science Center, Hiroshima University, Higashi-Hiroshima, \\ Hiroshima, Japan \\ ${ }^{3}$ Subaru Telescope, National Astronomical Observatory of Japan, Hilo, HI \\ ${ }^{4}$ Max-Planck Institut für Astrophysik, Karl-Schwarzschild-Strasse 2 D-85748 Garching bei \\ München, Germany \\ ${ }^{5}$ Istituto Naz. di Astrofisica-Oss. Astron., vicolo dell'Osservatorio, 5, 35122 Padova, Italy \\ ${ }^{6}$ Institute for the Physics and Mathematics of the Universe, University of Tokyo, Kashiwa, \\ Japan \\ ${ }^{7}$ Istituto Naz. di Astrofisica-Oss. Astron., Via Tiepolo, 11, 34131 Trieste, Italy \\ ${ }^{8}$ Department of Physical Science, Hiroshima University, Higashi-Hiroshima, Hiroshima, Japan
}

\begin{abstract}
Studying a multi-dimensional structure of supernovae ( $\mathrm{SNe}$ ) gives important constraints on the mechanism of the SN explosion. Polarization measurement is one of the most powerful methods to study the explosion geometry of extragalactic SNe. Especially, Type Ib/c $\mathrm{SNe}$ are the ideal targets because the core of the explosion is bare. We have performed spectropolarimetric observations of Type Ib/c SNe with the Subaru telescope. We detect a rotation of the polarization angle across the line, which is seen as a loop in the $Q-U$ plane. This indicates that axisymmetry is broken in the SN ejecta. Adding our new data to the sample of stripped-envelope SNe with high-quality spectropolarimetric data, five SNe out of six show a loop in the $Q-U$ plane. This implies that the SN explosion commonly has a non-axisymmetric, three-dimensional geometry.
\end{abstract}

Keywords. supernovae: general, techniques: polarimetric

\section{Introduction}

The explosion mechanism of supernovae (SNe) has been unclear for a long time after the first concept by Burbidge et al. (1957) and the first numerical simulation by Colgate \& White (1966). According to modern numerical simulations, a successful explosion cannot be obtained in one-dimensional simulations. And it is suggested that multi-dimensional effects, such as convection (e.g. Herant et al. (1994)) and Standing Accretion Shock Instability (SASI, e.g. Blondin et al. (2003)) are important.

Given these circumstances, it is important to obtain observational constraints on the multi-dimensional geometry of SN explosions. Spectropolarimetry is one of the most powerful methods to study the multi-dimensional geometry of extragalactic SNe (see Wang \& Wheeler (2008) for a review). To study the explosion geometry with spectropolarimetry, Type Ib/c SNe are the ideal targets because the core of the explosion is bare. We have performed spectropolarimetric observations of Type Ib/c SNe with the Subaru telescope. In these proceedings, we summarize spectropolarimetric properties of Type Ibc SNe. 


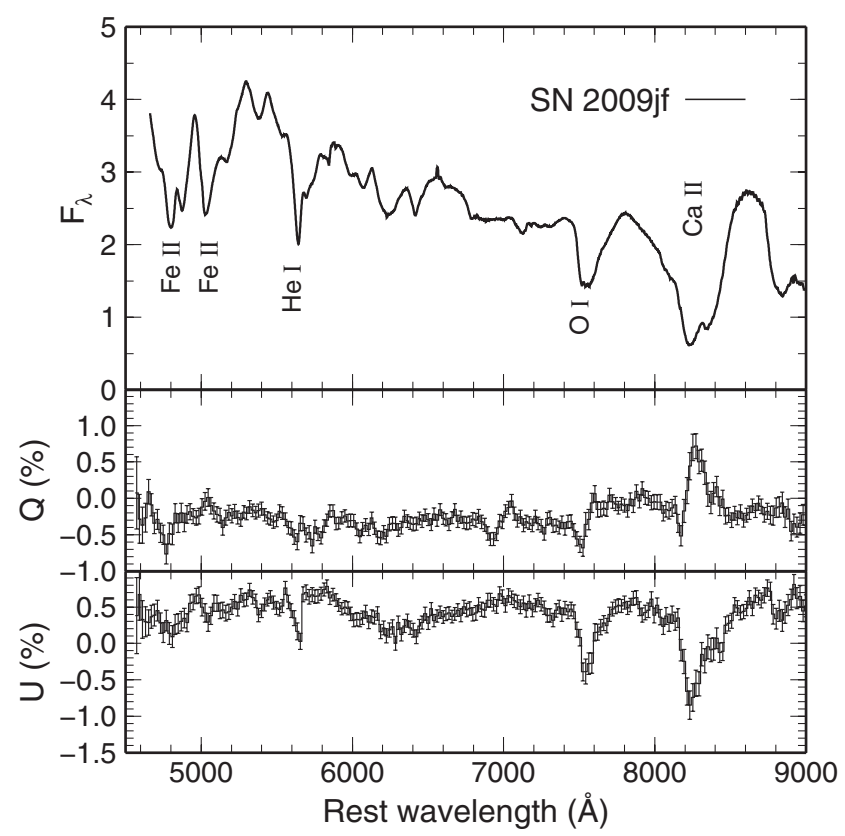

Figure 1. Total flux spectrum and polarization spectrum of SN 2009jf at 9.3 days after maximum. The total flux is shown in unit of $10^{-15} \mathrm{erg} \mathrm{s}^{-1} \mathrm{~cm}^{-2} \AA^{-1}$. For the polarization spectrum, the contribution of the interstellar polarization is not corrected for.

\section{Subaru Spectropolarimetry of SNe 2009jf and 2009mi}

We have performed spectropolarimetric observations of Type Ib SN 2009jf and Type Ic SN 2009mi with the Subaru telescope equipped with the Faint Object Camera and Spectrograph (FOCAS, Kashikawa et al. (2002)). The data for SNe 2009jf and 2009mi were obtained on UT 2009 October 24.3 (MJD = 55128.3) and 2010 January 8.3 (MJD=55204.3), respectively. These epochs correspond to 9.3 and 26.5 days after the $B$ band maximum $(\mathrm{MJD}=55118.96$ for SN 2009jf according to Sahu et al. $(2011)$, and MJD $=55177.8$ for SN 2009mi, based on our observations). More details of the observations are given in Tanaka et al. (2012).

Figure 1 shows the observed total flux and polarization spectrum of SN 2009jf. We clearly detect the changes in the polarization at the strong absorption lines, such as He I, O I, and Ca II. The data around the strong lines are shown in the $Q-U$ plane in Figure 2. It is clear that the data at the Ca II and O I lines occupy different regions in the $Q-U$ plane, indicating different spatial distributions between Ca II and O I. Such a difference is also clearly seen in other SNe e.g. Type Ib SN 2008D (Maund et al. (2009)) and Type IIb SN 2008ax (Chornock et al. (2011)).

A more interesting feature is the shape of the polarization data in the $Q-U$ plane. The Ca II and O I lines in SN 2009jf show a loop at these lines. This means that the polarization angle varies with Doppler velocity. As suggested by e.g. Kasen et al. (2003), Maund et al. (2007a), Maund et al. (2007b), this loop indicates that axisymmetry is broken in the SN ejecta. We also detect a similar (although less significant) loop in SN $2009 \mathrm{mi}$. Loops in the $Q-U$ plane have also been observed in Type Ia SNe (Wang et al. (2003a), Kasen et al. (2003), Chornock \& Filippenko (2008), Patat et al. (2009), Tanaka et al. (2010)). 

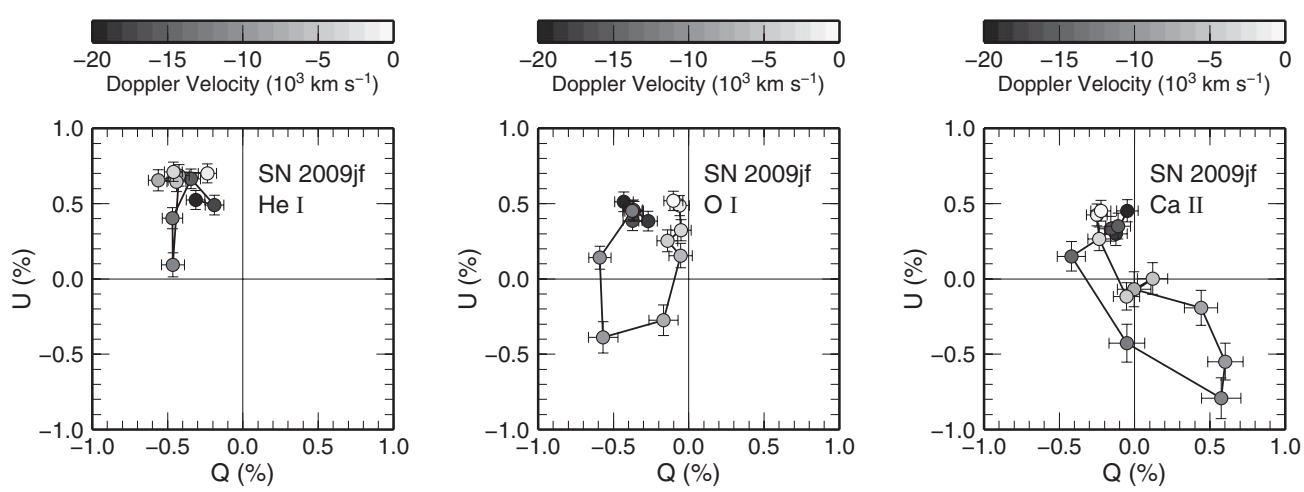

Figure 2. Polarization data of SN 2009jf in the $Q-U$ plane. Data around the He I (left), O I (center) and Ca II (right) lines are shown. Different data points show the polarization at different Doppler velocities as shown in the bar above the plots.

Table 1. Summary of Spectropolarimetric Observations of Type Ibc Supernovae

\begin{tabular}{|l|c|c|c|}
\hline Object & Type & Loop? & Reference \\
\hline SN 2005bf & Ib & yes & 1,2 \\
SN 2008D & Ib & yes & 3 \\
SN 2009jf & Ib & yes & 4 \\
\hline SN 2002ap & Ic broad & yes & $5,6,7$ \\
SN 2007gr & Ic & no & 8 \\
SN 2009mi & Ic & yes & 4 \\
\hline
\end{tabular}

References: ${ }^{1}$ Maund et al. (2007a), ${ }^{2}$ Tanaka et al. (2009a), ${ }^{3}$ Maund et al. (2009), ${ }^{4}$ Tanaka et al. (2012)

${ }^{5}$ Kawabata et al. (2002), ${ }^{6}$ Wang et al. (2003b), ${ }^{7}$ Leonard et al. (2002), ${ }^{8}$ Tanaka et al. (2008)

\section{Spectropolarimetric Properties of Type Ibc Supernovae}

In Table 1, we list up Type Ibc SNe with high-quality spectropolarimetric data. As also noted by Wang \& Wheeler (2008), all SNe show non-zero polarization, which means that stripped-envelope SNe generally have asymmetric explosion geometry. In addition, five $\mathrm{SNe}$ out of six show loops in $Q-U$ plane. This implies that a non-axisymmetric, 3D geometry is common in stripped-envelope SNe.

This is somewhat surprising since the line profiles in the nebular spectra, which are another probe of the explosion geometry, are nicely modelled by a bipolar geometry (e.g. Mazzali et al. (2001), Maeda et al. (2002), Mazzali et al. (2005), Maeda et al. (2008), Modjaz et al. (2008), Tanaka et al. (2009b), Taubenberger et al. (2009), Maurer et al. (2010), but see also Milisavljevic et al. (2010)). It must be noted, however, that spectropolarimetry is sensitive to the outer ejecta while nebular line profile is sensitive to the inner ejecta. Thus, for example, 3D perturbation onto a bipolar structure can also be consistent with the observations.

It must be interesting to look for a possible relation between spectropolarimetric properties and nebular line profiles. In fact, we did such an attempt, but could not find any significant correlation because of the small sample size (Tanaka et al. 2012). It is important to increase spectropolarimetric samples to further study fully multi-dimensional structure of SNe. 


\section{References}

Blondin, J. M., Mezzacappa, A., \& DeMarino, C. 2003, ApJ, 584, 971

Burbidge, E. M., Burbidge, G. R., Fowler, W. A., \& Hoyle, F. 1957, Reviews of Modern Physics, 29,547

Chornock, R. \& Filippenko, A. V. 2008, AJ, 136, 2227

Chornock, R., et al. 2011, ApJ, 739, 41

Colgate, S. A. \& White, R. H. 1966, ApJ, 143, 626

Herant, M., Benz, W., Hix, W. R., Fryer, C. L., \& Colgate, S. A. 1994, ApJ, 435, 339

Kasen, D., et al. 2003, ApJ, 593, 788

Kashikawa, N., et al. 2002, PASJ, 54, 819

Kawabata, K. S., et al. 2002, ApJ (Letters), 580, L39

Leonard, D. C., Filippenko, A. V., Chornock, R., \& Foley, R. J. 2002, PASP, 114, 1333

Maeda, K., Nakamura, T., Nomoto, K., Mazzali, P. A., Patat, F., \& Hachisu, I. 2002, ApJ, 565, 405

Maeda, K., et al. 2008, Science, 319, 1220

Maund, J. R., Wheeler, J. C., Patat, F., Baade, D., Wang, L., \& Höflich, P. 2007a, MNRAS, 381,201

Maund, J. R., Wheeler, J. C., Patat, F., Wang, L., Baade, D., \& Höflich, P. A. 2007b, ApJ, 671, 1944

Maund, J. R., Wheeler, J. C., Baade, D., Patat, F., Höflich, P., Wang, L., \& Clocchiatti, A. 2009, ApJ, 705, 1139

Maurer, J. I., et al. 2010, MNRAS, 402, 161

Mazzali, P. A., et al. 2005, Science, 308, 1284

Mazzali, P. A., Nomoto, K., Patat, F., \& Maeda, K. 2001, ApJ, 559, 1047

Milisavljevic, D., Fesen, R. A., Gerardy, C. L., Kirshner, R. P., \& Challis, P. 2010, ApJ, 709, 1343

Modjaz, M., Kirshner, R. P., Blondin, S., Challis, P., \& Matheson, T. 2008, ApJ (Letters), 687, L9

Patat, F., Baade, D., Höflich, P., Maund, J. R., Wang, L., \& Wheeler, J. C. 2009, A\&্A, 508, 229

Sahu, D. K., Gurugubelli, U. K., Anupama, G. C., \& Nomoto, K. 2011, MNRAS, 383

Tanaka, M., Kawabata, K. S., Maeda, K., Hattori, T., \& Nomoto, K. 2008, ApJ, 689, 1191

Tanaka, M., et al. 2009a, ApJ, 699, 1119

Tanaka, M., et al. 2009b, ApJ, 700, 1680

Tanaka, M., et al. 2010, ApJ, 714, 1209

Tanaka, M., et al. 2012, ApJ, submitted

Taubenberger, S., et al. 2009, MNRAS, 397, 677

Wang, L., et al. 2003a, ApJ, 591, 1110

Wang, L., Baade, D., Höflich, P., \& Wheeler, J. C. 2003b, ApJ, 592, 457

Wang, L. \& Wheeler, J. C. 2008, ARA\&A, 46, 433

\section{Discussion}

Couch: (Comment) SASI is not necessary to break axisymmetry. Bipolar magnetorotational explosions will break axisymmetry in 3D simulations.

CHORNock: I agree that $Q-U$ loops are likely a signature of large-scale structures in the ejecta, but how do you explain the different behaviors of different lines? Do we expect oxygen and calcium to have such different spatial distributions?

TANAKA: Oxygen is synthesized at the pre-SN stage while calcium can be synthesized by the explosion. So, qualitatively, these two elements can have different distributions in the SN ejecta. In fact, helium, which is also synthesized at the pre-SN stage, occupies a similar region to oxygen in the $Q-U$ plane in SN 2009jf. 